\title{
BMJ Open Relationship between metabolic syndrome and its components and cardiovascular disease in middle-aged and elderly Chinese population: a national cross-sectional survey
}

\author{
Wenzhen $\mathrm{Li},{ }^{1}$ Fujian Song, ${ }^{2}$ Xiaojun Wang, ${ }^{1}$ Dongming Wang, ${ }^{3}$ Dajie Chen, ${ }^{1}$ \\ Wei Yue, ${ }^{4}$ Hong Zhang, ${ }^{5}$ Zhihong Wang, ${ }^{6}$ Zuxun Lu ${ }^{1}$
}

To cite: Li W, Song F, Wang X, et al. Relationship between metabolic syndrome and its components and cardiovascular disease in middle-aged and elderly Chinese population: a national crosssectional survey. BMJ Open 2019;9:e027545. doi:10.1136/ bmjopen-2018-027545

- Prepublication history and additional material for this paper are available online. To view these files, please visit the journal online (http://dx.doi. org/10.1136/bmjopen-2018027545).

ZW and ZL contributed equally.

Received 27 October 2018

Revised 10 June 2019

Accepted 17 July 2019

Check for updates

(C) Author(s) (or their employer(s)) 2019. Re-use permitted under CC BY-NC. No commercial re-use. See rights and permissions. Published by BMJ.

For numbered affiliations see end of article.

Correspondence to

Professor Zuxun Lu;

zuxunlu@yahoo.com

\section{ABSTRACT}

Objectives To assess the relationship between metabolic syndrome (MetS) and its components and cardiovascular disease (CVD) according to different criteria of MetS, as well as whether the estimated association between MetS and CVD was affected by different definitions of MetS among the Chinese population.

Design Population-based, cross-sectional study. Setting Data were from a large-scale national stroke screening survey, China National Stroke Screening and Prevention Project.

Participants A nationally representative sample of 109551 Chinese adults aged $\geq 40$ years in 2014-2015 were included.

Primary outcome measures CVD conditions (stroke, coronary heart disease (CHD) and atrial fibrillation (AF)) diagnosed by clinicians were self-reported.

Results ORs after adjusting for CHD, stroke, AF and CVD in those with MetS using the National Cholesterol Education Program Adult Treatment Panel III (NCEP ATP III) criterion were $1.56(95 \% \mathrm{Cl} 1.48$ to 1.63$), 1.23(95 \% \mathrm{Cl}$ 1.17 to 1.30$), 1.14$ (95\% Cl 1.08 to 1.21$)$ and $1.40(95 \%$ Cl 1.35 to 1.45$) ; 1.51$ (95\% Cl 1.44 to 1.58$), 1.20$ (95\% Cl 1.14 to 1.26$), 1.09$ (95\% Cl 1.04 to 1.15$)$ and $1.34(95 \% \mathrm{Cl}$ 1.29 to 1.38) with the American Heart Association/National Heart, Lung, and Blood Institute criterion; and 1.41 (95\% Cl 1.35 to 1.48$), 1.24$ (95\% Cl 1.19 to 1.30$), 1.12$ (95\% $\mathrm{Cl} 1.06$ to 1.18$)$ and $1.31(95 \% \mathrm{Cl} 1.27$ to 1.35$)$ with the International Diabetes Federation criterion, respectively. Elevated blood pressures were all highly related to the prevalence of stroke and $\mathrm{AF}$, and reduced high-density lipoprotein-cholesterol was associated with a higher OR for CHD than other individual components of MetS.

Conclusions MetS is significantly associated with CVD, and the prevalence of CVD was more evident when MetS was defined according to the NCEP ATP III criterion. Developing effective public health strategies for the prevention, detection and treatment of MetS should be an urgent priority to reduce the burden of CVD in China.

\section{BACKGROUND}

Cardiovascular disease (CVD) mortality has been declining in most Western countries

\section{Strengths and limitations of this study}

- A nationally representative sample of 109551 Chinese adults were included, which increases the validity of the study.

- We compared three different definitions of metabolic syndrome (MetS) - the updated International Diabetes Federation, National Cholesterol Education Program Adult Treatment Panel III and American Heart Association/National Heart, Lung, and Blood Institute- to evaluate the association between MetS and coronary heart disease, stroke and atrial fibrillation.

- A limitation is the lack of longitudinal data, which could create causal inference.

since the 1980s. ${ }^{1-4}$ However, CVD morbidity and mortality have been increasing in China, ${ }^{5-8}$ with an estimated 21 million additional CVD cases by $2030 .^{9}$ Metabolic syndrome (MetS) refers to a cluster of cardiovascular risk factors, including abdominal obesity, elevated blood pressure (EBP), dyslipidaemia and dysglycaemia, which are associated with the development of $\mathrm{CVD}^{10}$ and increased risk of mortality from CVD and all causes. $^{11-13}$

Previous studies have explored the effects of MetS on the risk of CVD in different populations, but the results were inconsistent. A study based on two prospective cohorts ${ }^{14}$ showed that MetS and its components had weak or no associated risk of CVD in elderly populations using the National Cholesterol Education Program Adult Treatment Panel III (NCEP ATP III) definition, and a meta-analysis of 37 longitudinal studies showed only a modest relative risk among patients with MetS for CVD. ${ }^{15}$ However, MetS was associated with a twofold increase in age-adjusted 
risk for fatal CVD in men and non-fatal CVD in women in a Dutch population-based cohort study. ${ }^{16}$

Only a few population-based studies were conducted in China with regard to the association between MetS and CVD. A cross-sectional study ${ }^{17}$ suggested that MetS, as defined by the revised NCEP ATP III criterion, was associated with coronary heart disease (CHD). Another cross-sectional study ${ }^{18}$ of 2334 elderly in an urban community in Beijing in 2001-2002 showed that MetS was associated with the prevalence of CHD, stroke and peripheral arterial disease using the NCEP ATP III and the updated International Diabetes Federation (IDF) criteria.

MetS can be defined using different criteria, which inevitably led to substantial confusion and absence of comparability between studies. Different definitions also have their applicability to different ethnic groups, such as in terms of obesity cut-offs. ${ }^{19}$ However, it is still unclear which definition of MetS is the most suitable for use in the Chinese population. In the present study, we used data from a large-scale, national cross-sectional study to compare three different definitions of MetSupdated IDF, NCEP ATP III and American Heart Association/National Heart, Lung, and Blood Institute (AHA/ NHLBI) - to evaluate the association between MetS and CHD, stroke and atrial fibrillation (AF). We also investigated to what extent the individual components of MetS and their occurrence in pairs or triplets or quartets were associated with CVD in Chinese middle-aged and elderly populations, as well as whether the estimated association between MetS and CVD was affected by different definitions of MetS.

\section{METHODS}

\section{Study subjects and design}

We used data from a large-scale, national stroke screening survey, China National Stroke Screening and Prevention Project (CNSSPP). The survey was administrated by the National Project Office of Stroke Prevention and Control and carried out in 30 provinces (not including the province of Tibet) in China from October 2014 to November 2015; the rationale, design and methods have previously been described in detail. ${ }^{2021}$ Briefly, a total of 200 project areas were first selected in proportion to the local population size and the number of countries using a two-stage stratified cluster sampling method. Then an urban community and a rural village were selected from each project area as primary sampling units according to geographical locations and suggestions from local hospitals. The cluster sampling method was used in every primary sampling unit, and all residents aged $\geq 40$ years were surveyed during the primary screening. Questionnaire completion, physical examination and the assessment of risk factors of stroke were conducted in primary healthcare institutions, and the data were collected by trained medical staff using a standardised questionnaire in order to control for potential sources of bias. In addition, a sample of study participants were randomly selected in each primary sampling unit for further laboratory tests, carotid ultrasound and ECG. The analysis on MetS was restricted to individuals who had completed all surveys and examinations. A total of 726451 participants were included in the CNSSPP survey in the CNSSPP survey, and 109551 of them who received additional laboratory tests with a complete data on MetS and all the covariates were included in the present study. Participants could obtain the results from the primary healthcare institutions 2 weeks later.

\section{Definition of MetS}

The first definition of MetS was the updated IDF definition. The updated IDF defines MetS as central obesity (waist circumference $\geq 90 \mathrm{~cm}$ in men and $\geq 80 \mathrm{~cm}$ in women) plus any two of the following four additional factors: (1) hypertriglyceridaemia triglyceride level $\geq 1.7 \mathrm{mmol} / \mathrm{L}$; (2) high blood pressure $\geq 130 / 85 \mathrm{~mm} \mathrm{Hg}$ or treatment of previously diagnosed hypertension; (3) reduced high-density lipoprotein (HDL)-cholesterol $<1.03 \mathrm{mmol} / \mathrm{L}$ in men and $<1.29 \mathrm{mmol} / \mathrm{L}$ in women, or specific treatment for these lipid abnormalities; and (4) hyperglycaemia fasting glucose level of $\geq 5.6 \mathrm{mmol} / \mathrm{L}$ or treatment of previously diagnosed type 2 diabetes.

The second definition of MetS was the NCEP ATP III definition. A person was deemed to have MetS when three or more of the following five criteria were met: the criteria for high blood pressure, hypertriglyceridaemia and reduced HDL cholesterol were the same as those of the IDF, but the central obesity was defined as waist circumference $\geq 102 \mathrm{~cm}$ in men and $\geq 88 \mathrm{~cm}$ in women, and hyperglycaemia was defined as fasting glucose level of $\geq 6.1 \mathrm{mmol} / \mathrm{L}$ or treatment of previously diagnosed type 2 diabetes.

The third definition of MetS was the AHA/NHLBI definition. A person was considered to have MetS when three or more of the following five criteria were satisfied: waist circumference, high blood pressure, hypertriglyceridaemia and reduced HDL cholesterol were defined as those of the NCEP ATP III, but hyperglycaemia was defined as a fasting glucose level of $\geq 5.6 \mathrm{mmol} / \mathrm{L}$ or previously diagnosed type 2 diabetes.

\section{Diagnosis of CVD}

CVD conditions (stroke, CHD and AF) in the current study were based on participants' self-reports of these diseases as diagnosed by clinicians.

\section{Covariates}

Data on sociodemographic characteristics (age, gender, education and marital status), lifestyle, history of chronic diseases and physical activity were collected through face-to-face interviews by trained researchers. People who smoke at least one cigarette per day for more than half a year were defined as current smokers, and those who drink once or more per week for more than half a year were defined as current drinkers. Adequate physical activity was defined as regular exercise for more than three 
times per week and at least $30 \mathrm{~min}$ each time, or engaged in heavy physical work. Family history of chronic diseases diagnosed by a physician was self-reported, including hypertension, diabetes mellitus, CHD and stroke.

\section{Data analysis}

The t-test and $\chi^{2}$ test were used to analyse continuous and categorical variables, respectively. Logistic regressions were used to calculate ORs and their 95\% CIs. Potential confounders (age $(40-49,50-59, \geq 60)$, gender (male, female), marital status (married, single or divorced, widowed), level of education (primary school and below, middle school, high school or the equivalent, university or other tertiary degree), currently smoking (yes, no), currently drinking (yes, no), physical activity (yes, no), and family histories of CVD (yes, no) and diabetes mellitus (yes, no)) were adjusted. All analyses were conducted using SPSS V.19.0.

\section{Patient and public involvement}

This analysis is of secondary data collected 5 years ago, and accordingly the participants included were not involved in the conceptualisation or design of our specific study.

\section{RESULTS}

The present study used data from 109551 participants (aged $\geq 40$ years) who received clinical examinations. Table 1 shows the basic characteristics of the 109551 subjects (49789 men and 59762 women). Except for body mass index and fasting glucose, statistically significant differences were found between men and women in other demographic, anthropometric and clinical characteristics.

Table 2 shows the estimated association between different CVD conditions and MetS or components of MetS by different definitions. It showed in all MetS criteria that MetS was significantly associated with the prevalence of CHD, stroke and AF. However, the ORs for CVD associated with MetS defined by NCEP ATP III seemed to be stronger than those associated with MetS defined by other criteria. We also calculated the prevalence of CVD by sex, and the adjusted ORs of CVD based on MetS defined by NCEP ATP III criterion were 1.43 (95\% CI 1.35 to 1.51 ) for men and 1.39 (95\% CI 1.33 to 1.45 ) for women. Overall, the number of MetS components was positively associated with a higher prevalence of CVDs. A significantly higher prevalence of $\mathrm{CHD}$, stroke, $\mathrm{AF}$ and CVD with an increasing number of MetS components was also found based on the three criteria (figure 1).

The effects of various combinations of MetS traits on the three conditions according to the presence of a single component and their combination in pairs, triplets and quartets are shown in online supplementary table 1 . The risk for conditions associated with specific trait combinations was estimated with the group without that specific combination used as the comparator. The analysis suggested heterogeneity in the distribution of the various trait combinations that make up MetS and the associated heterogeneity in the risk for conditions. In terms of a single component from all criteria, low level of HDL cholesterol was associated with the highest OR for CHD (OR, 1.75, 95\% CI 1.67 to 1.83 ), and EBP was associated with the highest ORs for stroke (OR, 1.95, 95\% CI 1.84 to 2.07 ), $\mathrm{AF}$ (OR, $1.35,95 \% \mathrm{CI} 1.27$ to 1.43 ) and CVD (OR, 1.56, 95\% CI 1.50 to 1.62). The analyses based on different combinations of two, three and four MetS traits were more informative, and the results indicated that the ORs for CHD and CVD were higher in the combinations that included reduced HDL-cholesterol than other combinations, and the combinations that included EBP were all highly related to the ORs for stroke and AF.

\section{DISCUSSION}

In the present study, subjects with MetS defined by any of the three criteria had an increased prevalence of CHD and stroke. Many studies ${ }^{14}{ }^{15}$ have been conducted in other populations to examine the associations between MetS and CHD or stroke, and the results were controversial. Using the modified NCEP criteria, Shaista Malik et $a l^{22}$ showed CHD, CVD and total mortality were significantly higher in US adults aged 30-74 with MetS than in those without. However, results from the Prospective Study of Pravastatin in the Elderly at Risk (PROSPER) and British Regional Heart Study (BRHS) studies suggested weak or no association was found between MetS and vascular risk in elderly populations using the NCEP ATP III definition. ${ }^{14}$ The NCEP ATP III definition of MetS was associated with a twofold increase in age-adjusted risk for fatal CVD in men and non-fatal CVD in women in a Dutch population-based cohort study, and the cohort study also showed MetS was associated with different increased risks of incident cardiovascular morbidity and mortality in European population when different definitions were used. ${ }^{16}$ Thus, the results of the studies may be different when using different definitions of MetS or conducted in different populations. The results of our study were in compliance with a previous study conducted in a Chinese population, ${ }^{18}$ and the association seemed to be stronger in men than in women.

As far as we know, there are currently no convincing data on the association between MetS and AF in the Chinese population. AF is the most common arrhythmia in clinical practice and is associated with an increased risk of ischaemic stroke, heart failure and overall mortality. ${ }^{23-26}$ A recent study ${ }^{27}$ in 47 countries reported that the second highest number of stroke occurred in patients with $\mathrm{AF}$ in China. The components of MetS are also risk factors for the development of $\mathrm{AF}^{28}{ }^{29}$ A prospective, community-based cohort study in Japan showed that the association between MetS and AF remained significant in subjects without treated hypertension using the NCEP ATP III definition (HR, 1.78) but not using the AHA/NHLBI definition (HR, 1.28). ${ }^{30}$ Another study conducted in the USA suggested that in non-diabetic patients with essential 
Table 1 Demographic, anthropometric and plasma biochemical characteristics of subjects

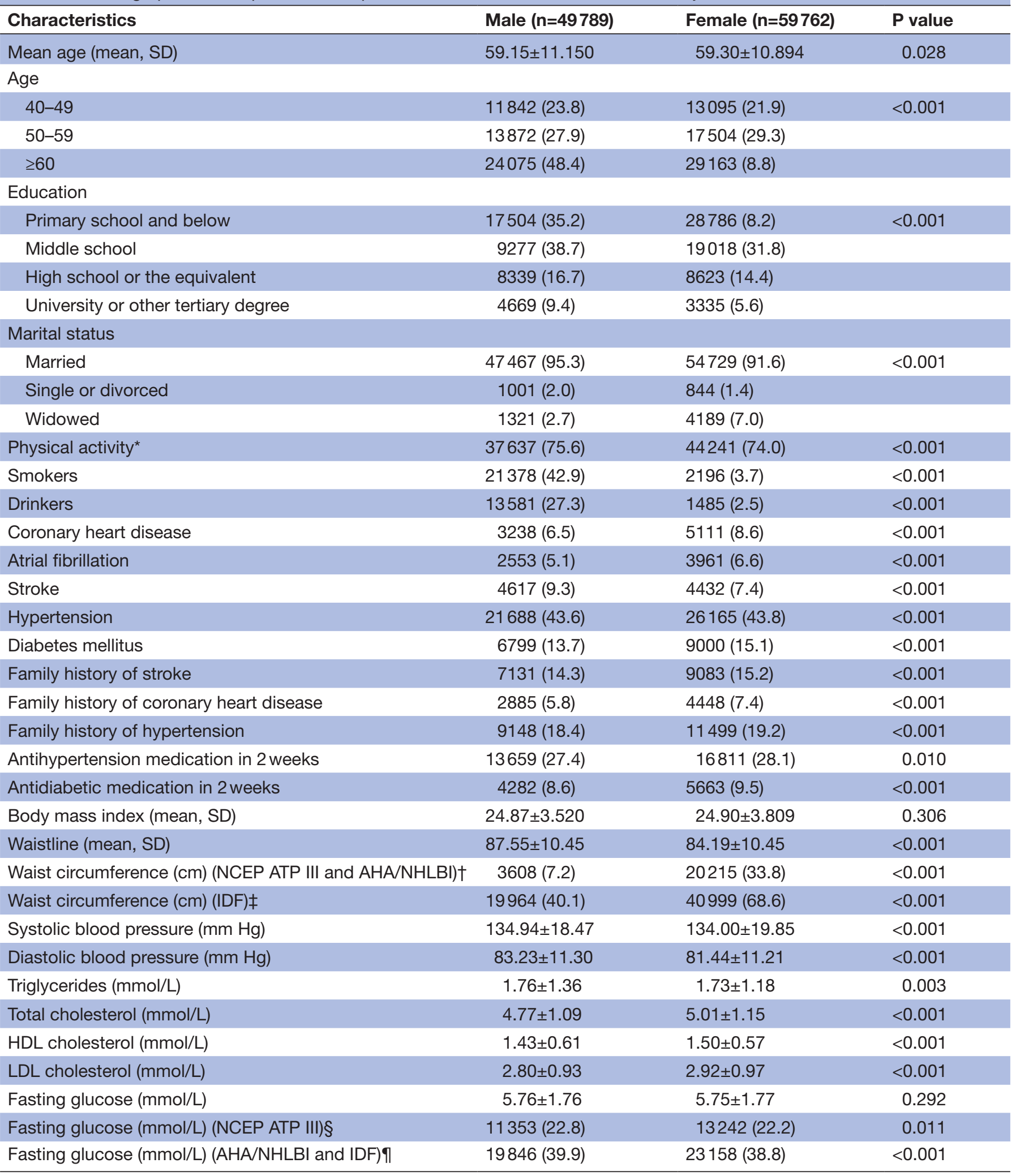

*Physical activity $\geq 3$ per week and $\geq 30$ min each time, or the person works in non-industrial/agriculture labour. †Waist circumference $\geq 102 \mathrm{~cm}$ in men and $\geq 88 \mathrm{~cm}$ in women.

$\ddagger$ Waist circumference $\geq 90 \mathrm{~cm}$ in men and $\geq 80 \mathrm{~cm}$ in women.

$\S$ Fasting glucose level of $\geq 5.6 \mathrm{mmol} / \mathrm{L}$.

qFasting glucose level of $\geq 6.1 \mathrm{mmol} / \mathrm{L}$.

AHA, American Heart Association; HDL, high-density lipoprotein; IDF, International Diabetes Federation; LDL, low-density lipoprotein; NCEP ATP III, National Cholesterol Education Program Adult Treatment Panel III; NHLBI, National Heart, Lung, and Blood Institute. 


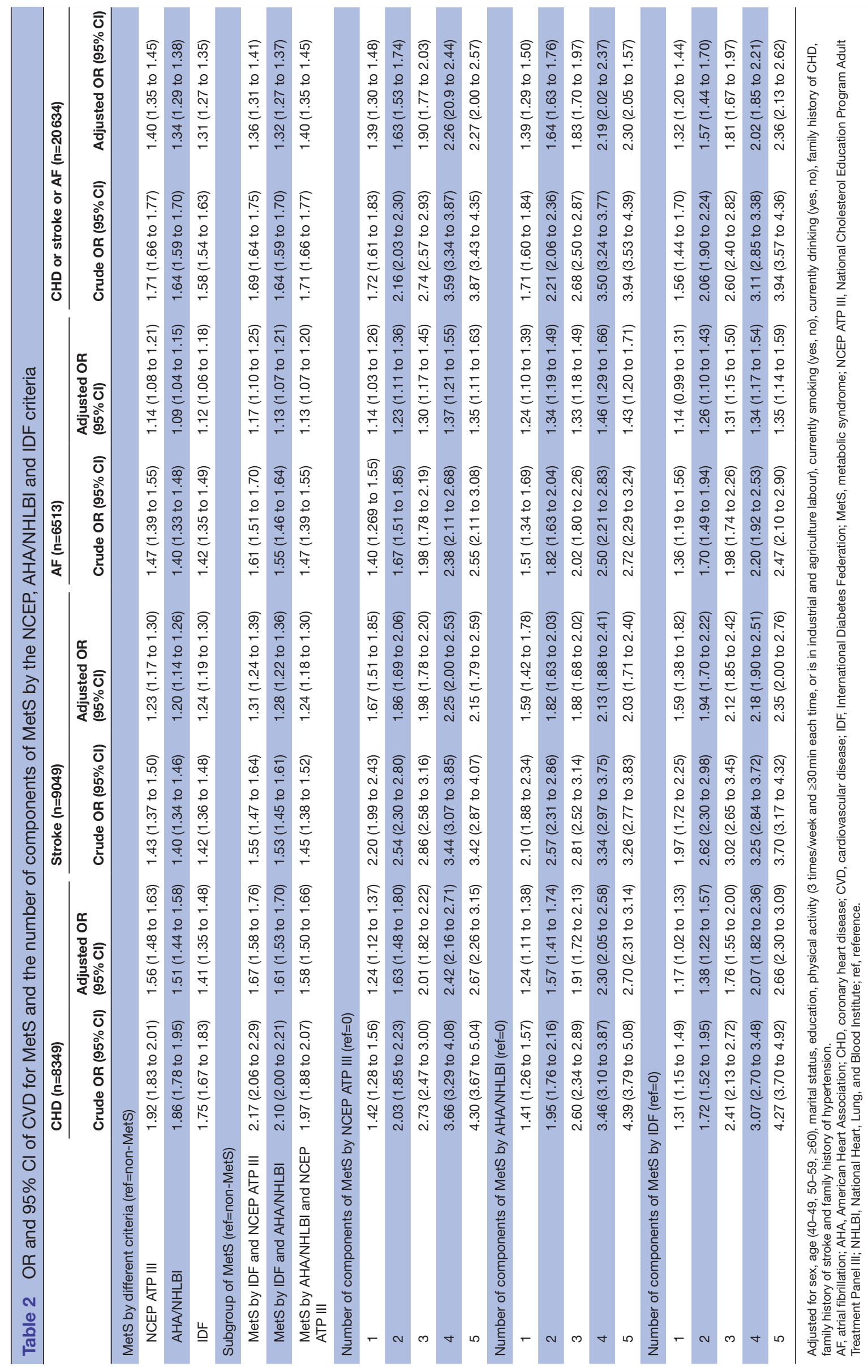




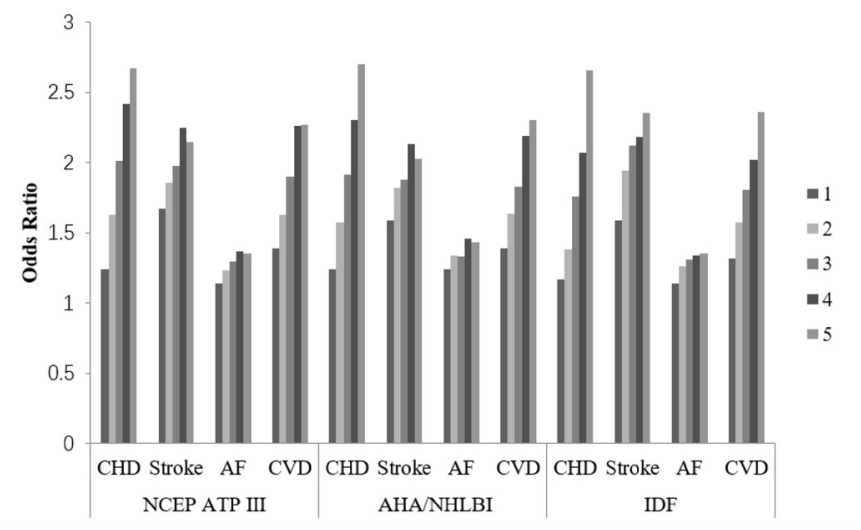

Figure 1 ORs of MetS with different numbers of components according to the three definitions of MetS among patients with $\mathrm{CHD}$, stroke or AF. AF, atrial fibrillation; AHA, American Heart Association; CHD, coronary heart disease; CVD, cardiovascular disease; IDF, International Diabetes Federation; MetS, metabolic syndrome; NCEP ATP III, National Cholesterol Education Program Adult Treatment Panel III; NHLBI, National Heart, Lung, and Blood Institute.

hypertension, MetS is directly and independently related to the prevalence of AF. Our study indicated that MetS was associated with the prevalence of AF using three definitions, which supported previous studies conducted in other countries.

In addition, we conducted further analyses to explore which component or combinations of MetS were associated with the three conditions. The results showed that subjects with low HDL cholesterol had the highest OR for CHD and individuals with EBP had the highest risk for stroke, AF and CVD. In addition, hypertriglyceridaemia was associated with an increased prevalence of $\mathrm{CHD}$, but not stroke or AF, and low HDL cholesterol was not associated with AF. The differences in associations of MetS traits with these diseases may be due to the different pathology mechanisms, and these findings suggested priority risk factors that should be considered for the prevention and management of different diseases. Moreover, we also found that subjects with different trait combinations had diverse prevalence of diseases. For example, subjects with low HDL cholesterol and hyperglycaemia (glucose $\geq 6.1 \mathrm{mmol} / \mathrm{L}$ ) had a higher OR for CHD than other pairs, and those with low HDL cholesterol and hyperglycaemia (glucose $\geq 6.1 \mathrm{mmol} / \mathrm{L}$ ) and hypertriglyceridaemia (and central obesity (waist $\geq 90 / 80 \mathrm{~cm}$ )) had the highest OR for CHD among triplets (quartets). These findings indicated that low HDL cholesterol was the most important risk factor for CHD. Similarly, EBP was an important risk factor for stroke and AF. In addition, our study also showed that more MetS components were associated with a higher risk of diseases. Thus, doctors and patients should pay more attention on the populations with multicomponents of MetS.

Several expert groups such as the IDF and the ATP III have proposed definitions of MetS. However, there is a lack of universally accepted definition around the world. $^{31}{ }^{32}$ Although all the existing definitions include obesity, hypertension, hyperglycaemia and dyslipidaemia, there are still differences in details and criteria, and controversy remains on how to define the cut-off points for each component of the cluster and the way of combining them. Therefore, it is necessary to examine the relationship between individual components of MetS or their cut-offs and CVD to develop a practical and generally accepted definition of MetS. Our study showed that the odds for CVD were higher when the NCEP ATP III definition was used than when the updated IDF or AHA/ NHLBI definition was used. Thus, the NCEP ATP III definition may be recommended to estimate the risk of CVD based on the present data. Our results were inconsistent with Frank B Hu's study, ${ }^{18}$ which indicated that the IDF criterion seemed to be better than the NCEP ATP III criterion. The different types of CVD may contribute to the explanation, as peripheral arterial disease was included as CVD in that study. Further studies that include more GVDs should be conducted in the future.

This study is a national study with a large sample size that aimed to examine the association between MetS and the prevalence of CHD and stroke, as well as the first study on the association between MetS and the prevalence of $\mathrm{AF}$ in the Chinese population, which will contribute to confirming the associations between MetS and different CVD conditions. It is reported that AF will become an increasingly significant burden in developing countries, and the prevalence of $\mathrm{AF}$ in those aged over 60 was estimated to rise from 3.9 million to 9 million by 2050 in China. ${ }^{33}$ Our study revealed that the risk of AF may be deceased by $10 \%$ if MetS could be prevented and EBP controlled. In addition, our study also indicated that low HDL cholesterol and EBP had the strongest associations with the risk of CVD, and findings on the relationships between MetS individual component and their combinations in pairs or triplets or quartets and the risks of these diseases will be important for disease prevention and clinical treatment. As we know, different definitions of MetS inevitably result in substantial confusion and absence of comparability between studies. Our study compares the differences of the ORs of MetS with CVD using different definitions and cut-offs, and we believe it is helpful in developing a generally accepted definition of MetS.

Some limitations of the study are worth mentioning. First of all, the study is a cross-sectional study and data could not be used for causal inference, which is a main limitation in epidemiological studies in general. Second, the diseases were self-reported, which may lead to misclassification and underestimation of the association of MetS with the risk of diseases. However, previous studies have found that the associations of morbidity and mortality with self-reported $\mathrm{AF}$ were similar to those with electrocardiographically detected $\mathrm{AF}^{34}{ }^{35}$ In addition, although our analyses were conducted adjusting for smoking and alcohol consumption, some other lifestyle factors were still not included. Also, HDL cholesterol, triglyceride and blood glucose levels were measured only once, which 
might be imprecise and result in random errors. Furthermore, the present study included only participants who had additional laboratory tests, and the association of MetS and CVD may be overestimated because high-risk CVD was more prevalent among them. Last but not least, the WHO definition and that of the European Group for the Study of Insulin Resistance were not used in the present study to define MetS for lack of assessment of impaired glucose or insulin resistance, which requires a more complicated test.

In summary, our findings indicate that MetS is significantly associated with CVD in all the three definitions of MetS. The prevalence of CVD was more evident when MetS was defined according to the NCEP ATP III criterion, compared with the updated IDF and AHA/NHLBI criteria. Among middle-aged and elderly Chinese population, low HDL cholesterol and EBP are more prevalent than other components of MetS for ORs of CVD. Strategies for the prevention and treatment of MetS should be developed and implemented to reduce the socioeconomic and medical burden of CVD in China.

\section{Author affiliations}

${ }^{1}$ Department of Social Medicine and Health Management, School of Public Health, Tongji Medica College, Huazhong University of Science and Technology, Wuhan, China

${ }^{2}$ Norwich Medical School, Faculty of Medicine and Health Science, University of East Anglia, Norwich, UK

${ }^{3}$ Department of Occupational and Environmental Health, School of Public Health, Tongji Medica College, Huazhong University of Science and Technology, Wuhan, China

${ }^{4}$ Neurology Department, Tianjin Huanhu Hospital, Tianjin, China

${ }^{5}$ Department of Science and Education, People's Hospital of Deyang City, Deyang, China

${ }^{6}$ Health Science Center, Shenzhen University First Affiliated Hospital, Shenzhen, China

Contributors WY and HZ helped conduct the survey and build the data set. WL analysed the data and wrote the draft of the paper. DW, XW, DC and FS revised and edited the paper. DW and XW contributed to the discussion. ZW and ZL are the guarantors.

Funding This work was supported by the Ministry of Finance of the People's Republic of China (issued by Finance and Social Security (2011) Document No 61, Ministry of Finance), the Fundamental Research Funds for the Central Universities, Huazhong University of Science and Technology, Wuhan, China (2016YXMS215), the Innovation Committee of Shenzhen Science and Technology (KJYY20170413162318686), and the Fundamental Research Funds for the Central Universities (2019kfyXJJS032). "2018 National Social Science Fund "Research on the Construction of China's Graded Medical System under the Shared Economy" (18ZDA085)".

Competing interests None declared.

Patient consent for publication Not required.

Ethics approval The study protocol was approved by the Ethics Committee of Xuan Wu Hospital, Capital Medical University (Beijing, China) (approval ID (2015)024). All the respondents were provided with the informed consent form and were informed that they had the right to participate or refute the investigation. All questionnaires were filled in by respondents anonymously.

Provenance and peer review Not commissioned; externally peer reviewed.

Data availability statement Data may be obtained from a third party and are not publicly available.

Open access This is an open access article distributed in accordance with the Creative Commons Attribution Non Commercial (CC BY-NC 4.0) license, which permits others to distribute, remix, adapt, build upon this work non-commercially, and license their derivative works on different terms, provided the original work is properly cited, appropriate credit is given, any changes made indicated, and the use is non-commercial. See: http://creativecommons.org/licenses/by-nc/4.0/.

\section{REFERENCES}

1. Feigin VL, Forouzanfar MH, Krishnamurthi R, et al. Global and regional burden of stroke during 1990-2010: findings from the global burden of disease study 2010. The Lancet 2014;383:245-55.

2. Moran $\mathrm{AE}$, Forouzanfar MH, Roth GA, et al. Temporal trends in ischemic heart disease mortality in 21 world regions, 1980 to 2010: the global burden of disease 2010 study. Circulation 2014;129:1483-92.

3. Mundal L, Igland J, Ose L, et al. Cardiovascular disease mortality in patients with genetically verified familial hypercholesterolemia in Norway during 1992-2013. Eur J Prev Cardiol 2017;24:137-44.

4. Shay CM, Ning H, Allen NB, et al. Status of cardiovascular health in US adults: prevalence estimates from the National health and nutrition examination surveys (NHANES) 2003-2008. Circulation 2012;125:45-56.

5. He J, Gu D, Wu X, et al. Major causes of death among men and women in China. N Engl J Med 2005;353:1124-34.

6. Liu M, Wu B, Wang W-Z, et al. Stroke in China: epidemiology, prevention, and management strategies. Lancet Neurol 2007;6:456-64.

7. Yang G, Wang Y, Zeng Y, et al. Rapid health transition in China, 1990-2010: findings from the global burden of disease study 2010. The Lancet 2013;381:1987-2015.

8. Zhang X-H, Lu ZL, Liu L. Coronary heart disease in China. Heart 2008;94:1126-31.

9. Moran A, Gu D, Zhao D, et al. Future cardiovascular disease in China: Markov model and risk factor scenario projections from the coronary heart disease policy model-china. Circ Cardiovasc Qual Outcomes 2010;3:243-52.

10. Haffner SM, Valdez RA, Hazuda HP, et al. Prospective analysis of the insulin-resistance syndrome (syndrome X). Diabetes 1992;41:715-22.

11. Isomaa B, Almgren P, Tuomi T, et al. Cardiovascular morbidity and mortality associated with the metabolic syndrome. Diabetes Care 2001;24:683-9.

12. Lakka H-M, Laaksonen DE, Lakka TA, et al. The metabolic syndrome and total and cardiovascular disease mortality in middle-aged men. JAMA 2002;288:2709-16.

13. Sundström J, Risérus U, Byberg L, et al. Clinical value of the metabolic syndrome for long term prediction of total and cardiovascular mortality: prospective, population based cohort study. BMJ 2006;332:878-82.

14. Sattar N, McConnachie A, Shaper AG, et al. Can metabolic syndrome usefully predict cardiovascular disease and diabetes? outcome data from two prospective studies. The Lancet 2008;371:1927-35.

15. Gami AS, Witt BJ, Howard DE, et al. Metabolic syndrome and risk of incident cardiovascular events and death: a systematic review and meta-analysis of longitudinal studies. J Am Coll Cardiol 2007:49:403-14.

16. Dekker JM, Girman C, Rhodes T, et al. Metabolic syndrome and 10-year cardiovascular disease risk in the Hoorn study. Circulation 2005;112:666-73.

17. Lu J, Wang L, Li M, et al. Metabolic syndrome among adults in China: the 2010 China noncommunicable disease surveillance. J Clin Endocrinol Metab 2017;102:507-15.

18. He Y, Jiang B, Wang J, et al. Prevalence of the metabolic syndrome and its relation to cardiovascular disease in an elderly Chinese population. J Am Coll Cardiol 2006;47:1588-94.

19. Appropriate body-mass index for Asian populations and its implications for policy and intervention strategies. The Lancet 2004;363:157-63.

20. Wang X, Li W, Song F, et al. Carotid atherosclerosis detected by ultrasonography: a national Cross-Sectional study. J Am Heart Assoc 2018;7.

21. Li W, Song F, Wang X, et al. Prevalence of metabolic syndrome among middle-aged and elderly adults in China: current status and temporal trends. Ann Med 2018;50:345-53.

22. Malik S, Wong ND, Franklin SS, et al. Impact of the metabolic syndrome on mortality from coronary heart disease, cardiovascular disease, and all causes in United States adults. Circulation 2004;110:1245-50.

23. Fedeli U, Ferroni E, Pengo V. Mortality associated to atrial fibrillation still on the rise: United States, 1999 to 2014. Int J Cardiol 2016;222:788-9. 
24. Benjamin EJ, Wolf PA, D'Agostino RB, et al. Impact of atrial fibrillation on the risk of death: the Framingham heart study. Circulation 1998;98:946-52.

25. Lloyd-Jones DM, Wang TJ, Leip EP, et al. Lifetime risk for development of atrial fibrillation: the Framingham heart study. Circulation 2004;110:1042-6.

26. Wolf PA, Abbott RD, Kannel WB. Atrial fibrillation as an independent risk factor for stroke: the Framingham study. Stroke 1991;22:983-8.

27. Healey JS, Oldgren J, Ezekowitz M, et al. Occurrence of death and stroke in patients in 47 countries 1 year after presenting with atrial fibrillation: a cohort study. The Lancet 2016;388:1161-9.

28. Kannel WB, Wolf PA, Benjamin EJ, et al. Prevalence, incidence, prognosis, and predisposing conditions for atrial fibrillation: population-based estimates. Am J Cardiol 1998;82:2N-9.

29. Movahed M-R, Hashemzadeh M, Jamal MM. Diabetes mellitus is a strong, independent risk for atrial fibrillation and flutter in addition to other cardiovascular disease. Int J Cardiol 2005;105:315-8.
30. Watanabe H, Tanabe N, Watanabe T, et al. Metabolic syndrome and risk of development of atrial fibrillation: the Niigata preventive medicine study. Circulation 2008;117:1255-60.

31. Expert Panel on Detection, Evaluation, and Treatment of High Blood Cholesterol in Adults. Executive summary of the third report of the National cholesterol education program (NCEP) expert panel on detection, evaluation, and treatment of high blood cholesterol in adults (adult treatment panel III). JAMA 2001;285:2486-97.

32. Alberti KGMM, Zimmet P, Shaw J. The metabolic syndrome-a new worldwide definition. The Lancet 2005;366:1059-62.

33. Hajhosseiny R, Matthews GK, Lip GYH. Metabolic syndrome, atrial fibrillation, and stroke: tackling an emerging epidemic. Heart Rhythm 2015;12:2332-43.

34. Psaty BM, Manolio TA, Kuller LH, et al. Incidence of and risk factors for atrial fibrillation in older adults. Circulation 1997;96:2455-61.

35. Furberg CD, Psaty BM, Manolio TA, et al. Prevalence of atrial fibrillation in elderly subjects (the cardiovascular health study). Am J Cardiol 1994;74:236-41. 